F.-L.Deubner, M.Reichling and R.Langhanki

Institut für Astronomie und Astrophysik, Universität Würzburg Am Hubland, 8700 Würzburg, F.R.G.

EXTENDED ABSTRACT. The amount of energy carried in the solar atmosphere by short period acoustic waves is particularly uncertain in the range of frequencies $(\nu \geqslant 10 \mathrm{mHz})$ which is potentially relevant for acoustic heating.

Utilizing the diagnostic of phase diagrams, the existence of coherent short period wave trains has been demonstrated (Deubner et al.,1984). The same technique which was previously introduced to correct for systematic phase shifts caused by seeing, can be applied to extract from a weak and noisy signal the solar part for frequencies higher than $10 \mathrm{mHz}$.

The apparent Doppler shift amplitudes measured in the cross power spectra are then compared with those of synthetic solar line profiles to yield estimates of the energy carried by short period waves at various photospheric / chromospheric levels.

At a height of $\sim 300 \mathrm{~km}$ in the gtmosphere we observe a mechanical energy flux of $\sim 1.010^{7}$ erg $\mathrm{cm}^{-2} \mathrm{~s}^{-1}$.

The following effects have been considered in deducing intrinsic wave amplitudes from the observed Doppler shifts in solar spectral lines as a function of frequency:

a) SEEING

Seeing is the dominant source of noise in the power spectra of velocity/ brightness fluctuations: Image motion (as well as stochastic instrumental guiding errors) cause an erratic scanning of the solar image. Thus the large amplitude background fields (p-mode oscillations) become the source of a spurious high frequency signal. Itscontribution to the measured signal may be identified and estimated quantitatively by comparing the fluctuations in different spectral lines.

Depending on the lines chosen these spurious signals are either in phase or in antiphase, yielding at high frequencies a distribution of relative phases in the complex cross spectra which scatter around $0^{\circ}$ or $180^{\circ}$. The frequency $\nu^{*}$ where the imaginary part of the mean amplitude vanishes determines the limit up to which a useful solar signal may be 
extracted from the data (cf. Fig. 1).
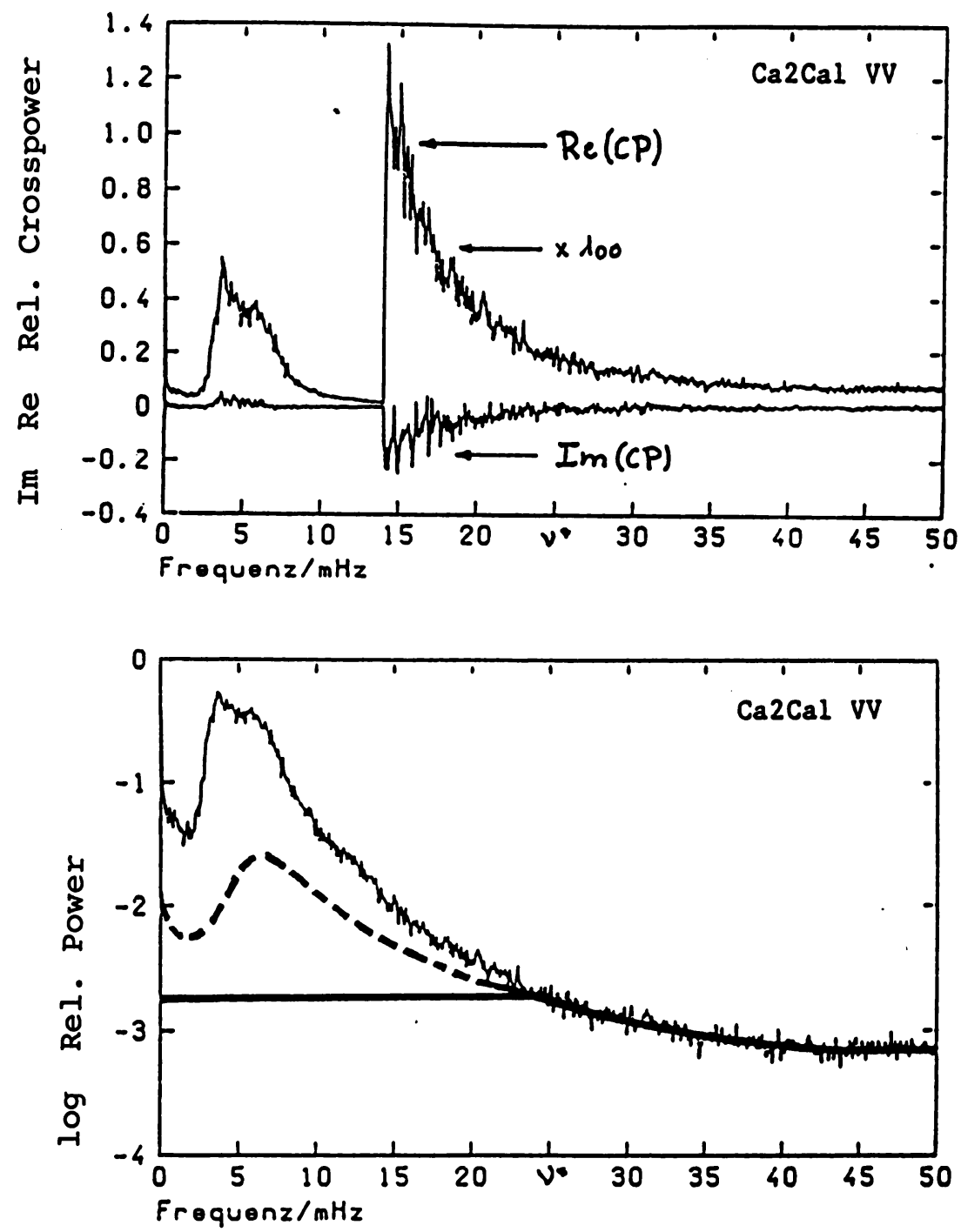

Fig. 1. Above: The real and imaginary part of cross covariance amplitudes of velocity fluctuations in the Ca 8542 and Ca 8498 lines as functions of frequency. At $\nu^{*}=25 \mathrm{mHz}$ the imaginary part vanishes, indicating the non-solar origin of power (in the real part) at higher frequencies. Correction for seeing noise is accomplished by subtracting real amplitudes from the complex cross power spectra, as indicated by the heavy solid line in the lower part of the Figure.

\section{b) PROPAGATION}

The $k$ - $\boldsymbol{\omega}$ diagram of spatially resolved data is utilized to separate the propagating and the non-propagating compone nts of the wave field. The 
resulting $\boldsymbol{\nu}$-dependent reduction factor is then applied to the one-dimensional frequency spectra. This procedure defines a lower limit of the observed acoustic power because seeing noise which cannot be removed from the $k-\omega$ spectra diminishes the factor.

On the other hand, $V-V$ and $V-I$ phase spectra both indicate the presence of genuine standing waves which do not contribute to the net energy flow.

c) OBLIQUITY OF WAVES

The measured amplitude of obliquely running wave trains is $\propto \cos \theta$ which compensates for the effect of the obliquity on the radial flow magnitude. Small scale disturbances are likely to be partially missed in the cross power signal due to their reduced vertical coherence.

d) OPTICAL FILTERING IN THE LINE FORMING LAYER

In a kinematic simulation a realistic solar atmosphere was perturbed with small amplitude short period wave trains to evaluate the filtering effect of the finite width of the line forming layer on high frequency wave amplitudes. The wavelength shift of the emerging line profiles was compared with the input wave amplitude to obtain the frequency dependent filtering factors or Modulation Transfer Function (MTF).

Finally, the MTF has been applied to the one-dimensional cross power spectrum produced in step b) to obtain estimates of the actual acoustic power as a function of frequency (Fig. 2). The integral of this function yields a mechanical energy flow defined as $F_{\text {mech }}=\rho c v_{\text {rms }}{ }^{2}$ of $1.710^{7} \mathrm{erg} \mathrm{cm}^{-2} \mathrm{~s}^{-1}$.

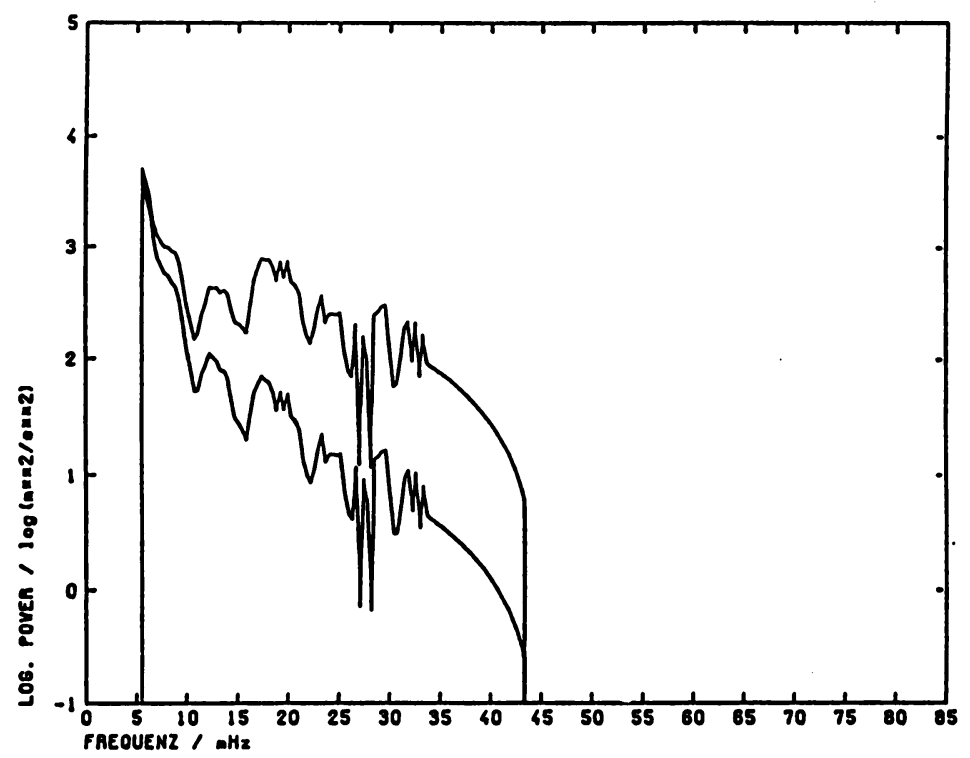

Fig. 2. Mean cross covariance amplitudes of coherent Doppler signals $V(F e$ 5383) - V(Ti 5381): after correction for seeing and evanescent waves (lower curve) and after correction with the MTF defined in step d). 
e) ASSESSMENT OF REMAINING SYSTEMATIC ERRORS

* Inspection of the phase distribution spectra suggests the presence of a genuine standing wave component (cf.e.g.Deubner et al., 1984) leading to an overestimate of the overall propagating acoustic power.

* The assumption of constant seeing noise power for $\nu<\nu^{*}$ like the solid line in Fig. $1 \mathrm{~b}$ entails another slight overestimate. (A more sophisticated noise power estimate is indicated by the dashed line.)

Both effects together will probably change the final result by a factor of two at most.

Further effects are likely to lead rather to an underestimate:

* The cutoff at $\nu^{*}$ introduced in step a). By extrapolating the monotonic decrease of the final power spectrum towards higher frequencies one can easily see the comparatively minor consequences of this cutoff.

* In step b), it is not feasible like in step a) to remove the seeing noise from the two-dimensional power spectra. Again, the magnitude of this effect is rather negligeable.

* Evanescent waves at very high wave numbers may be aliased into the propagating regime of the $k-\boldsymbol{\omega}$ diagram. However, as before the monotonic decrease of power towards higher wave numbers shows that the effect of such contamination cannot be large.

* In a realistic horizontally inhomogeneous atmosphere considerable scatter of the observed phases is anticipated which causes a decrease of the mean amplitudes at higher frequencies, irrespective of the power that is actually transmitted. This effect may in part explain the rapid dropping away of the corrected power spectra.

Taking all the arguments together, we suggest the value of $F_{\text {mech }}=$ $1.010^{7} \mathrm{erg} \mathrm{cm} \mathrm{cm}^{-1}$ as our safest estimate of the acoustic energy flux of the quiet sun at a level of approximately $300 \mathrm{~km}$ above $\tau_{5000}=1$. The evaluation of the results of other combinations of spectral lines formed at various heights in the atmosphere is in progress.

\section{References}

Deubner,F.-L., Endler,F., Staiger,J.: 1984, Mem.S.A.It. 5 55, 135. 